\title{
The 812-Carat Pure Type IaB Constellation Diamond from Karowe - Part of an Even Larger Rough?
}

\author{
Ulrika F.S. D’Haenens-Johansson, Evan M. Smith, Karen V. Smit, \\ Wuyi Wang, Thomas M. Moses \\ Gemological Institute of America, New York City, NY, USA, \\ ujohansson@gia.edu,evan.smith@gia.edu,ksmit@gia.edu,wwang@gia.edu,tmoses@gia.edu
}

\section{Introduction}

Lucara Diamond extracted an 812-carat colorless diamond from the AK6 pipe at Karowe mine (Botswana) in November 2015. Named "The Constellation," it is recognized as the sixth-largest gemquality rough diamond ever discovered. The Constellation attained the highest price for a rough diamond to date, selling in May 2016 to Dubai-based Nemesis International DMCC for \$63.1 million (\$77,649 per carat) (Graff 2016). Interestingly, several very large diamonds were recovered at Karowe within the same week. This group includes the second-largest gem-quality rough diamond ever found, the 1,109-carat colorless Lesedi La Rona, as well as other colorless diamonds weighing 374, 296, and 183 carats (Lucara Diamond 2016; Graff 2016; Shor 2016). The Constellation and the 296-carat and 183-carat diamonds were investigated at GIA using a combination of gemological and spectroscopic techniques to analyze the diamonds' point defect content. Evidence will be presented demonstrating that these diamonds are not unrelated, but stem from a single rough diamond that was shattered into multiple pieces.

\section{Sample and Experimental Details}

The Constellation and the 296-carat and 183-carat rough diamonds were separately submitted to our laboratory for scientific examination in 2016, prior to any cutting or polishing (see Figure 1). Their external morphologies and internal features were studied under magnification using Gem Instruments and Nikon SMZ1500 binocular microscopes using dark field and fiber optic illumination.

The diamonds' fluorescence behavior to ultraviolet (UV) illumination was tested using a combination gem lamp (365 nm and $254 \mathrm{~nm}$ emission) commonly used by the diamond industry. UV fluorescence imaging is a useful tool to reveal growth zones, which typically contain different concentrations of impurities reflecting growth fluid changes. A DiamondView fluorescence instrument $(<230 \mathrm{~nm})$ was used to image portions of the 183-carat and 296-carat stones - the large sizes restricted the possible viewing orientations in the chamber. The Constellation was imaged using a GIA-built system under illumination from a deuterium lamp $(185-400 \mathrm{~nm})$.
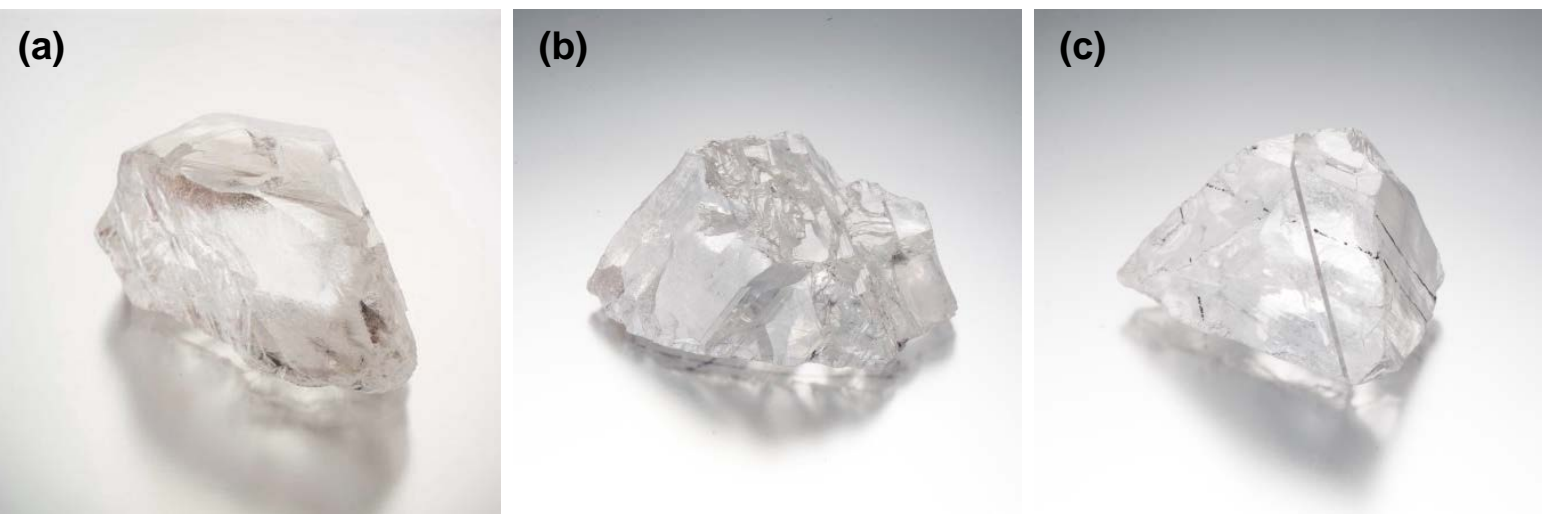

Figure 1: (a) The 812-carat Constellation (812.77 ct, $64 \times 39 \times 34 \mathrm{~mm})$, the (b) 296-carat $(183.41 \mathrm{ct}, 38 \times 23 \times$ $23 \mathrm{~mm}$ ), and (c) 183-carat diamonds originating from Karowe. Both (b) and (c) show ink markings in preparation for cutting. Photographs by Jiaxin (Jae) Liao. 
Impurity concentrations were analyzed using room temperature Fourier Transform Infrared (FTIR) spectroscopy (Thermo Nicolet Nexus 6700 spectrometer) and photoluminescence (PL) at $77 \mathrm{~K}$ using a range of excitation sources (Renishaw confocal microspectrometer with laser excitation wavelengths of 488.0, 514.5, 632.5, and $830.0 \mathrm{~nm}$ ).

\section{Results and Discussion}

Diamonds are commonly classified according to their nitrogen content measured by FTIR spectroscopy: Type I diamonds contain nitrogen in either isolated (Ib) or aggregated (IaAB) forms, while Type II diamonds do not contain detectable nitrogen concentrations (IIa), but may contain boron (IIb). Over the past five years at GIA, however, spectroscopic analyses of thousands of Type $\mathrm{IaB}$, IIa, and IIb colorless diamonds have revealed trends that demonstrate that a defect content transition can often be detected between diamond types, rather than being clearly separate groups, with Type IIb and pure IaB bracketing Type IIa diamonds. Based on inclusions, many Type IIa as well as some IaB diamonds originate from the sublithospheric mantle, in association with metallic liquid (Smith et al. 2016). Inclusions also indicate a "superdeep," sublithospheric mantle origin for Type IIb diamonds (Smith and Wang, 11IKC-4502).

All three diamonds in this study are pure Type IaB, containing $20 \pm 4$ ppm B-centers $\left(\mathrm{N}_{4} \mathrm{~V}\right)$. The diamonds studied here are notable for being the largest pure Type IaB samples reported, whereas large colorless diamonds are often highlighted as Type IIa. To emphasize the rarity of Type IaB diamonds, we analyzed 5,060 colorless to faintly colored (D-Z) faceted diamonds weighing between 10.0 and 228.3 carats that were submitted for grading services at GIA. As expected, the most common diamond type was Type IaAB (73.8\%). However, only $1.2 \%$ of diamonds were pure Type IaB, significantly rarer than Type IIa diamonds (24.6\%).

PL spectroscopy detected emission from $\mathrm{H} 4$ (496 nm, thought to be $\mathrm{N}_{4} \mathrm{~V}_{2}$ ), $\mathrm{H} 3\left(503.2 \mathrm{~nm}, \mathrm{NVN}^{0}\right.$ ) and GR1 (741 nm, $\mathrm{V}^{0}$ ) centers, consistent with natural Type IaB diamonds. Remarkably similar peak characteristics were observed among the suite, with comparable peak intensities and full-width halfmaxima (FWHM), as shown in Figure 2. Typically natural diamonds show highly variable PL peak characteristics and combinations, such that the distinctively similar FTIR and PL results in these three diamonds provide compelling evidence that they were once part of a single diamond.
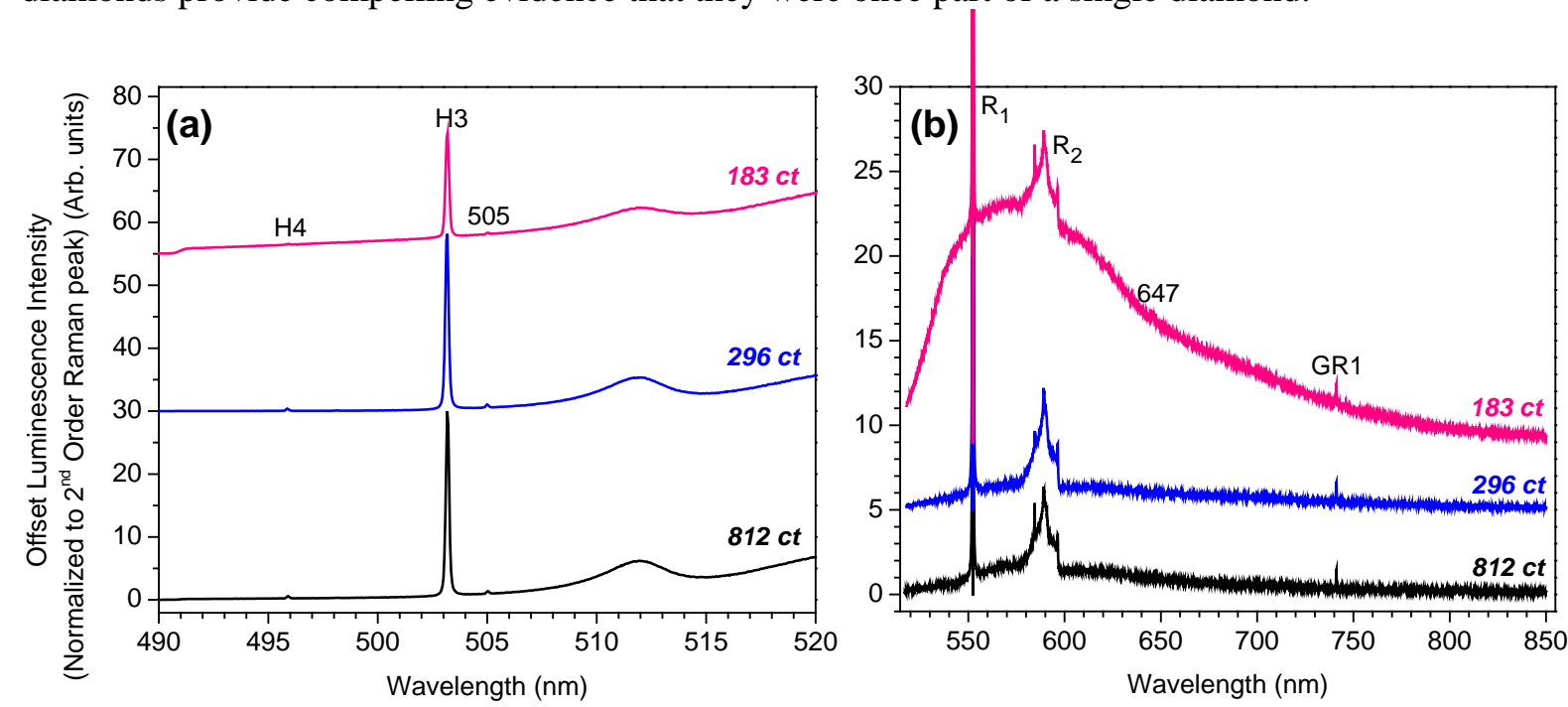

Figure 2: PL spectra collected at $77 \mathrm{~K}$ using (a) $488.0 \mathrm{~nm}$ and (b) $514.2 \mathrm{~nm}$ laser excitations. Data have been normalized to the corresponding $2^{\text {nd }}$-order diamond Raman peaks and offset for clarity. Spectra indicate low concentrations of luminescent defects, compared to the majority of diamonds investigated by GIA. Consistent emission from H4 (496 nm), H3 (503.2 nm) and GR1 (741 nm) centers were detected for all three diamonds. PL spectra obtained using $632.5 \mathrm{~nm}$ and $830.0 \mathrm{~nm}$ lasers (not shown) did not reveal any additional defect centers. In (b) $\mathrm{R}_{1}$ and $\mathrm{R}_{2}$ indicate the $1^{\text {st }}$ - and $2^{\text {nd }}$-order Raman peaks. FWHM for the H3 and GR1 emission peaks were calculated for each data set, and were found to be $0.181 \pm 0.006 \mathrm{~nm}$ and $0.36 \pm 0.02 \mathrm{~nm}$, respectively. 


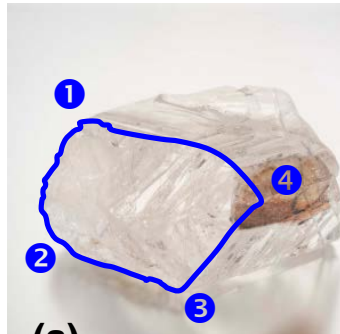

(a)

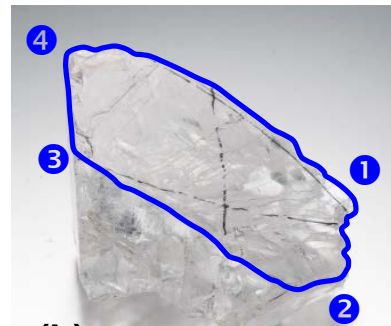

(b)

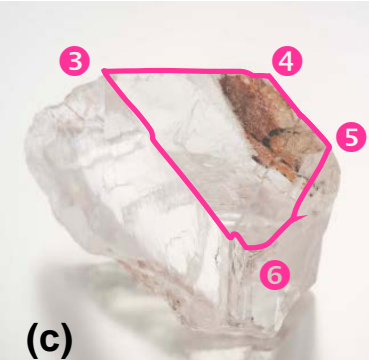

(c)

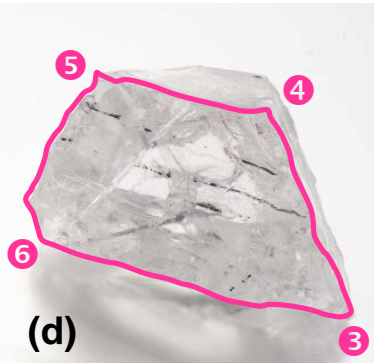

Figure 3: Images illustrating the common cleavage faces for (a, c) The Constellation and the (b) 296-carat and (c) 183-carat diamonds, with corners numbered to facilitate orientation. Outlines for the matching fractured surfaces are color-coded, with blue lines used for The Constellation and the 296-carat diamonds (a-b), and pink used for The Constellation and the 183-carat diamonds (c-d). The edge spanning labels 3 and 4 is common to all three stones. Photographs by Jiaxin (Jae) Liao.

The diamonds showed identical responses when exposed to the gem lamp: weak blue fluorescence, followed by weak blue phosphorescence to short-wave UV, and medium blue fluorescence to longwave UV. Fluorescence imaging revealed that each sample contained at least two distinct growth zones.

The external morphologies of the stones showed primary octahedral, resorbed, and fractured faces, with both The Constellation and 296-carat diamonds featuring distinct fractures containing metallic inclusions and secondary iron-oxide staining. Minor iron-oxide staining was also observed in the 183carat stone. The specimens were generally inclusion-free, with only a few small black or reddishorange inclusions spanning 200-500 $\mu \mathrm{m}$ in size being observed close to fractured surfaces. Detailed inspection of their surfaces revealed shared cleavage planes between The Constellation and the 296-carat and 183-carat stones. Outlines for the matching fractured surfaces are highlighted in Figure 3. Significantly, the iron-oxide stained fractures in The Constellation and the 296-carat diamonds converge to a paired corner (labeled 4). Furthermore, the intrinsic growth structures revealed by UV-fluorescence for these specific surfaces were consistent for the two diamonds. The visual observations further support the conclusion that these three specimens originate from the same rough, which may have broken either during kimberlite eruption or mining processes.

With a combined weight of 1,291 carats, the composite of the studied diamonds would have surpassed the Lesedi La Rona. In fact, due to the similar visual characteristics and extraction dates, it is possible that all five large Karowe diamonds originated from the same rough, 2774 carats combined, although this cannot be confirmed without further inspection. Nevertheless, even without considering the unexamined Lesedi La Rona and 374-carat diamonds, this report shows how multi-technique analyses of point defects and their distributions can link multiple rough diamonds comprising a single diamond of remarkable historic and scientific significance.

\section{References}

Graff M (2016) Lucara sells 812-carat diamond for \$63M. National Jeweler website. http://www.nationaljeweler.com/diamonds-gems/pricing/4220-lucara-sells-812-carat-diamond-for63m. Accessed 20 December 2016

Lucara Diamond (2016) April 2016 Exceptional Stone Tender Catalogue, Karowe Mine, Botswana Production. Lucara Diamond website. https://www.lucaradiamond.com/assets/docs/catalogues/ lucara-bonas-sdt9-apr132016-1476742115.pdf. Accessed 23 December 2016

Shor R (2016) Is another 1,000 (or 3,000) carat diamond around the corner? GIA website. https://www.gia.edu/gia-news-research/is-another-1000-carat-diamond-around-corner. Accessed 20 December 2016

Smith EM, Shirey SB, Nestola F, Bullock ES, Wang J, Richardson SH, Wang W (2016) Large gem diamonds from metallic liquid in Earth's deep mantle. Science 354(6318):1403-1405 doi:10.1126/science.aal1303 